Marquette University

e-Publications@Marquette

12-1-2009

\title{
Reactivity of phenyldi(2-thienyl)phosphine towards Group 7 Metal Carbonyls: Carbon-phosphorus Bond Activation
}

\author{
Shishir Ghosh \\ Jahangirnagar University \\ Alok K. Das \\ Jahangirnagar University \\ Noorjahan Begum \\ Lund University \\ Daniel T. Haworth \\ Marquette University \\ Sergey Lindeman \\ Marquette University, sergey.lindeman@marquette.edu
}

See next page for additional authors

Follow this and additional works at: https://epublications.marquette.edu/chem_fac

Part of the Chemistry Commons

\section{Recommended Citation}

Ghosh, Shishir; Das, Alok K.; Begum, Noorjahan; Haworth, Daniel T.; Lindeman, Sergey; Gardinier, James R.; Siddiquee, Tasneem A.; Bennett, Dennis W.; Nordlander, Ebbe; Hogarth, Graeme; and Kabir, Shariff E., "Reactivity of phenyldi(2-thienyl)phosphine towards Group 7 Metal Carbonyls: Carbon-phosphorus Bond Activation" (2009). Chemistry Faculty Research and Publications. 543.

https://epublications.marquette.edu/chem_fac/543 


\section{Authors}

Shishir Ghosh, Alok K. Das, Noorjahan Begum, Daniel T. Haworth, Sergey Lindeman, James R. Gardinier, Tasneem A. Siddiquee, Dennis W. Bennett, Ebbe Nordlander, Graeme Hogarth, and Shariff E. Kabir 
Marquette University

e-Publications@Marquette

\section{Department of Chemistry Faculty Research and Publications/College of Arts and Sciences}

This paper is NOT THE PUBLISHED VERSION.

Access the published version at the link in the citation below.

Inorganica Chimica Acta, Vol. 362, No. 15 (December 1, 2009): 5175-5182. DOI. This article is (C) Elsevier and permission has been granted for this version to appear in e-Publications@Marquette.

Elsevier does not grant permission for this article to be further copied/distributed or hosted elsewhere without the express permission from Elsevier.

\section{Reactivity of Phenyldi(2-thienyl)phosphine Towards Group 7 Metal Carbonyls: Carbon- Phosphorus Bond Activation}

\section{Shishir Ghosh}

Department of Chemistry, Jahangirnagar University, Savar, Dhaka-1342, Bangladesh

Alok K. Das

Department of Chemistry, Jahangirnagar University, Savar, Dhaka-1342, Bangladesh

Noorjahan Begum

Inorganic Chemistry Research Group, Chemical Physics, Center for Chemistry and Chemical Engineering, Lund University, Lund, Sweeden

Daniel T. Haworth

Department of Chemistry, Marquette University, Milwaukee, WI, USA

Sergey V. Lindeman

Department of Chemistry, Marquette University, Milwaukee, WI, USA

James F. Gardinier 
Department of Chemistry, Marquette University, Milwaukee, WI, USA

\section{Tanseem A. Siddiquee}

Department of Chemistry, University of Wisconsin-Milwaukee, Milwaukee, WI, USA

\section{Dennis W. Bennett}

Department of Chemistry, University of Wisconsin-Milwaukee, Milwaukee, WI, USA

\section{Ebbe Norlander}

Inorganic Chemistry Research Group, Chemical Physics, Center for Chemistry and Chemical Engineering, Lund University, Lund, Sweeden

\section{Graeme Hogarth}

Department of Chemistry, University College London, London, UK

\section{Shariff E. Kabir}

Department of Chemistry, Jahangirnagar University, Savar, Dhaka-1342, Bangladesh

\section{Abstract}

Addition of phenyldi(2-thienyl)phosphine $\left(\mathrm{PPhTh}_{2}\right)$ to $\left[\operatorname{Re}_{2}(\mathrm{CO})_{10-n}(\mathrm{NCMe})_{n}\right](n=1,2)$ affords the substitution products $\left[\operatorname{Re}_{2}(\mathrm{CO})_{10-n}\left(\mathrm{PhPTh}_{2}\right)_{n}\right](\mathbf{1}, \mathbf{2})$ together with small amounts of fac$\left[\mathrm{ClRe}(\mathrm{CO})_{3}\left(\mathrm{PPhTh}_{2}\right)_{2}\right](\mathbf{3})(n=2)$. Reaction of $\left[\operatorname{Re}_{2}(\mathrm{CO})_{10}\right]$ with $\mathrm{PPhTh}_{2}$ in refluxing xylene affords a mixture which includes 2, $\left[\operatorname{Re}_{2}(\mathrm{CO})_{7}\left(\mathrm{PPhTh}_{2}\right)(\mu-\mathrm{PPhTh})(\mu-\mathrm{H})\right](4)$, $\left[\operatorname{Re}_{2}(\mathrm{CO})_{7}\left(\mathrm{PPhTh}_{2}\right)(\mu-\mathrm{PPhTh})\left(\mu-\eta^{1}, \mathrm{~K}^{1}(\mathrm{~S})-\right.\right.$ $\left.\left.\mathrm{C}_{4} \mathrm{H}_{3} \mathrm{~S}\right)\right](5)$ and $m e r-\left[\mathrm{HRe}(\mathrm{CO})_{3}\left(\mathrm{PPhTh}_{2}\right)_{2}\right](\mathbf{6})$. Phosphido-bridged $\mathbf{4}$ and $\mathbf{5}$ are formed by the carbonphosphorus bond cleavage of the coordinated $\mathrm{PPhTh}_{2}$ ligand, the cleaved thienyl group being retained in the latter. Reaction of $\left[\mathrm{Mn}_{2}(\mathrm{CO})_{10}\right]$ with $\mathrm{PPhTh} 2$ in refluxing toluene affords $\left[\mathrm{Mn}_{2}(\mathrm{CO})_{9}\left(\mathrm{PPhTh}_{2}\right)\right](7)$ and the carbon-phosphorus bond cleavage products $\left[\mathrm{Mn}_{2}(\mathrm{CO})_{6}(\mu-\mathrm{PPhTh})\left(\mu-\eta^{1}, \eta^{5}-\mathrm{C}_{4} \mathrm{H}_{3} \mathrm{~S}\right)\right](8)$ and $\left[\mathrm{Mn}_{2}(\mathrm{CO})_{5}(\mathrm{PPhTh})(\mu-\mathrm{PPhTh})\left(\mu-\eta^{1}, \eta^{5}-\mathrm{C}_{4} \mathrm{H}_{3} \mathrm{~S}\right)\right](\mathbf{9})$. Both 8 and $\mathbf{9}$ contain a bridging thienyl ligand which is bonded to one manganese atom in a $\eta^{5}$-fashion.

\section{Graphical abstract}

Reactions of the dirhenium and dimanganese complexes $\left[\operatorname{Re}_{2}(\mathrm{CO})_{10-n}(\mathrm{NCMe})_{n}\right](n=0,1,2)$ and $\left[\mathrm{Mn}_{2}(\mathrm{CO})_{10}\right]$ with phenyldi(2-thienyl)phosphine have been investigated. A number of mono- and dinuclear complexes are obtained from these reactions by carbon-phosphorus bond cleavage of the phosphine ligand.
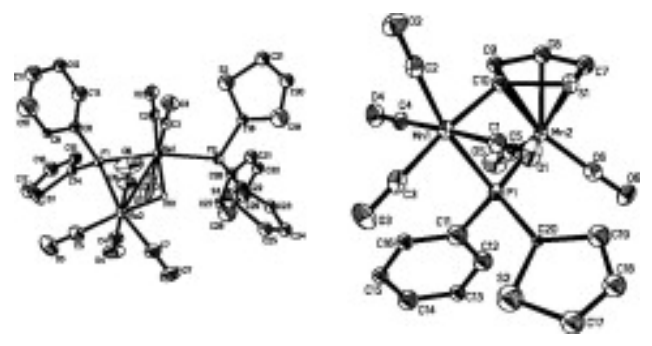


\section{Keywords}

Rhenium carbonyl, Manganese carbonyl, Phenyldi(2-thienyl)phosphine, Carbon-phosphorus bond cleavage, X-ray structures

\section{Introduction}

The coordination chemistry of thiophene and its derivatives with transition metal complexes has been extensively explored to understand the nature of the important reactions involved in metal-catalyzed hydrodesulfurization (HDS), a process that removes sulfur from the organosulfur impurities that are commonly found in liquid fuels [1], [2], [3], [4], [5], [6], [7], [8], [9], [10], [11], [12], [13], [14], [15], [16]. Numerous examples of thiophene coordination and activation have demonstrated that thiophene and its benzo-derivatives are not useful as ligands for transition metals, even for soft metals that normally bind strongly to sulfur. To overcome this disadvantage, thienyl phosphines have been used to introduce the heterocycle into the metal coordination sphere [17], [18], [19], [20], [21], [22], [23], [24], [25].

The reactivity of metal carbonyls of the iron triad with thienyl phosphines such as $\mathrm{Ph}_{2} \mathrm{PTh}(\mathrm{Th}=2$ thienyl) [18], [19], [20], [21], PhPTh 2 [22], diphenyl(benzothienyl)phosphine [22] and PTh 3 [23], [24] is well-documented, and a wealth of intriguing reactivity patterns have been observed. For example, we have recently shown that carbon-sulfur bond scission of a coordinated PTh 3 ligand at a triruthenium center leads to the formation of a $\mu_{3}-\eta^{3}$-1-thia-butadiene ligand [26]. In contrast, the reactivity of group 7 metal carbonyls with thienyl phosphines has not been as extensively studied. Deeming et al. have investigated the reactivity of $\mathrm{Ph}_{2} \mathrm{PTh}$ with $\left[\mathrm{Re}_{2}(\mathrm{CO})_{10}\right]$ and $\left[\mathrm{Mn}_{2}(\mathrm{CO})_{10}\right]$ [25] which leads to a number of interesting products (Chart 1). The dirhenium complex $\left[\operatorname{Re}_{2}(\mathrm{CO})_{8}\left(\mu-\mathrm{K}^{1}, \mathrm{~K}^{1}-\mathrm{Ph}_{2} \mathrm{PC}_{4} \mathrm{H}_{3} \mathrm{~S}\right)\right](\mathbf{A})$ results from the photochemical reaction with $\left[\operatorname{Re}_{2}(C O)_{10}\right]$, while in refluxing xylene the isomeric carbon-phosphorus bond cleavage product $\left[\operatorname{Re}_{2}(\mathrm{CO})_{8}\left(\mu-\mathrm{PPh}_{2}\right)\left(\mu-\eta^{1}: \mathrm{K}^{1}(\mathrm{~S})-\mathrm{C}_{4} \mathrm{H}_{3} \mathrm{~S}\right)\right](\mathrm{B})$ results. With $\left[\mathrm{Mn}_{2}(\mathrm{CO})_{10}\right]$ the reaction proceeds differently and in refluxing xylene a mixture of $\left[\mathrm{Mn}_{2}(\mathrm{CO})_{9}\left(\mathrm{Ph}_{2} \mathrm{PTh}\right)\right]$ (C) and $\left[\mathrm{Mn}_{2}(\mathrm{CO})_{6}\left(\mu-P P h_{2}\right)\left(\mu-\eta^{1}: \eta^{5}-\mathrm{C}_{4} \mathrm{H}_{3} \mathrm{~S}\right)\right]$ (D) are obtained. We recently reported the reactivity of $\mathrm{PTh}_{3}$ with the lightly stabilized dirhenium complexes $\left[\mathrm{Re}_{2}(\mathrm{CO})_{10-n}(\mathrm{NCMe})_{n}\right](n=0,1,2)$ and $\left[\mathrm{Mn}_{2}(\mathrm{CO})_{10}\right]$ [27]. With rhenium carbonyls, a series of mono- and dirhenium complexes were obtained $\left(\mathbf{E}-\mathbf{M}\right.$, Chart 2) while with $\left[\mathrm{Mn}_{2}(\mathrm{CO})_{10}\right.$ ] products $\mathbf{N}-\mathbf{P}$ (Chart 2) are similar to those reported with $\mathrm{Ph}_{2} \mathrm{PTh}$. For comparison, we have now investigated the reactivity of $\mathrm{PhPTh}_{2}$ with group 7 metal carbonyls, details of which are reported herein. 


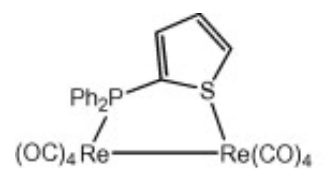

(A)

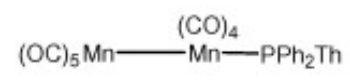

(C)

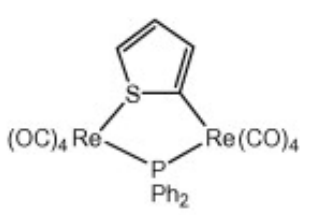

(B)

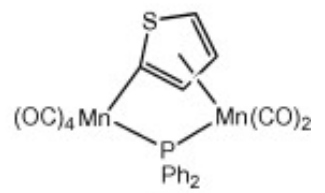

(D)

Chart 1.

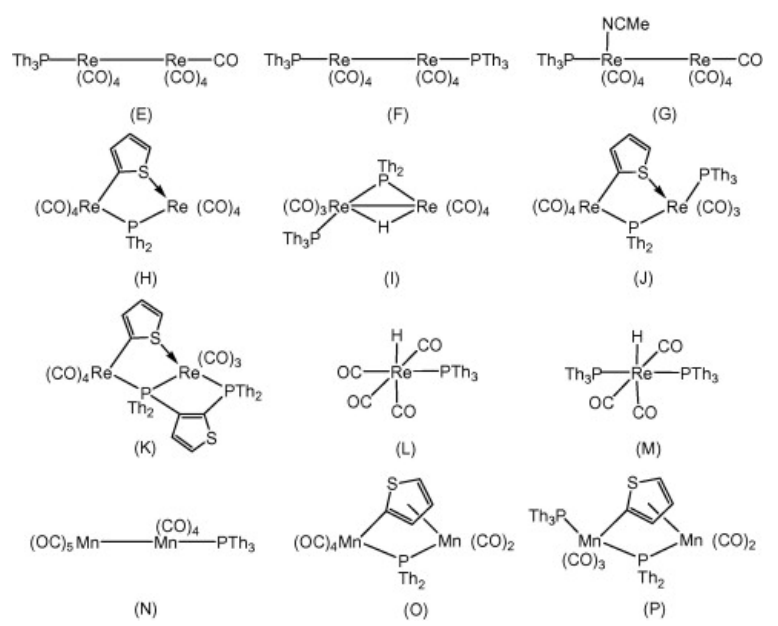

Chart 2.

\section{Experimental}

$\left[\mathrm{Re}_{2}(\mathrm{CO})_{10}\right]$ and $\left[\mathrm{Mn}_{2}(\mathrm{CO})_{10}\right]$ were purchased from Strem Chemicals Inc. and used without further purification, and $\left[\operatorname{Re}_{2}(\mathrm{CO})_{9}(\mathrm{NCMe})\right]$ and $\left[\mathrm{Re}_{2}(\mathrm{CO})_{8}(\mathrm{NCMe})_{2}\right]$ were prepared according to published procedures [28]. Phenyldi(2-thienyl)phosphine ( $\mathrm{PPhTh}$ ) was purchased from E. Merck and used as received. All reactions were carried out under a nitrogen atmosphere using standard Schlenk techniques. Reagent grade solvents were dried by the standard procedures and were freshly distilled prior to use. Infrared spectra were recorded on a Shimadzu FTIR 8101 spectrophotometer. NMR spectra were recorded on Bruker DPX 400 and Varian Inova 500 instruments. Elemental analyses were performed by Microanalytical Laboratories, University College London. Fast atom bombardment mass spectra were obtained on a JEOL SX-102 spectrometer using 3-nitrobenzyl alcohol as matrix and CSI as calibrant.

\subsection{Reaction of [Re2(CO)9(NCMe)] with PPhTh2}

A CH${ }_{2} \mathrm{Cl}_{2}$ solution $(30 \mathrm{~mL})$ of $\left[\mathrm{Re}_{2}(\mathrm{CO})_{9}(\mathrm{NCMe})\right](100 \mathrm{mg}, 0.150 \mathrm{mmol})$ and $\mathrm{PPhTh}_{2}(42 \mathrm{mg}, 0.153 \mathrm{mmol})$ was stirred at $25^{\circ} \mathrm{C}$ for $90 \mathrm{~h}$. The solvent was removed by rotary evaporation and the residue separated by TLC on silica gel. Elution with hexane $/ \mathrm{CH}_{2} \mathrm{Cl}_{2}(7: 3, \mathrm{v} / \mathrm{v})$ developed three bands. The first and second bands gave unreacted $\left[\operatorname{Re}_{2}(\mathrm{CO})_{10}\right]$ (trace) and $\mathrm{PPhTh}_{2}$ (trace), respectively, while the third band afforded $\left[\operatorname{Re}_{2}(\mathrm{CO})_{9}(\mathrm{PPhTh} 2)\right](1)(116 \mathrm{mg}, 84 \%)$ as pale yellow crystals after recrystallization from 
hexane/ $\mathrm{CH}_{2} \mathrm{Cl}_{2}$ at $4{ }^{\circ} \mathrm{C}$. Spectral data for 1: Anal. Calc. for $\mathrm{C}_{23} \mathrm{H}_{11} \mathrm{O}_{9} \mathrm{PRe}_{2} \mathrm{~S}_{2}: \mathrm{C}, 30.73 ; \mathrm{H}, 1.23$. Found: $\mathrm{C}$, 30.96; $\mathrm{H}, 1.30 \%$. IR ( $\left.v \mathrm{CO}, \mathrm{CH}_{2} \mathrm{Cl}_{2}\right): 2110 \mathrm{~s}, 2039 \mathrm{sh}, 2009 \mathrm{vs}, 1947 \mathrm{~s} \mathrm{~cm}^{-1} ;{ }^{1} \mathrm{H} \mathrm{NMR}\left(\mathrm{CDCl}_{3}\right): \delta 7.72(\mathrm{~m}$, 4H), $7.58(\mathrm{~m}, 2 \mathrm{H}), 7.48(\mathrm{~m}, 3 \mathrm{H}), 7.23(\mathrm{~m}, 2 \mathrm{H}) ;{ }^{31} \mathrm{P}-\left\{{ }^{1} \mathrm{H}\right\} \mathrm{NMR}\left(\mathrm{CDCl}_{3}\right): \delta-15.6(\mathrm{~s}) ; \mathrm{MS}(\mathrm{FAB}): \mathrm{m} / z 898\left(\mathrm{M}^{+}\right)$.

\subsection{Reaction of $[\mathrm{Re} 2(\mathrm{CO}) 8(\mathrm{NCMe}) 2]$ with $\mathrm{PPhTh} 2$}

To a $\mathrm{CH}_{2} \mathrm{Cl}_{2}$ solution $\left(30 \mathrm{~mL}\right.$ ) of $\left[\mathrm{Re}_{2}(\mathrm{CO})_{8}(\mathrm{NCMe})_{2}\right]$ (100 mg, $\left.0.147 \mathrm{mmol}\right), \mathrm{PPhTh}_{2}(81 \mathrm{mg}, 0.295 \mathrm{mmol})$ was added and the reaction mixture was stirred at $25^{\circ} \mathrm{C}$ for $72 \mathrm{~h}$. The solvent was removed under reduced pressure and the resultant residue was subjected to TLC on silica gel. Elution with hexane/ $\mathrm{CH}_{2} \mathrm{Cl}_{2}(7: 3, \mathrm{v} / \mathrm{v})$ developed four bands. The first and second bands gave unreacted $\left[\operatorname{Re}_{2}(\mathrm{CO})_{8}(\mathrm{NCMe})_{2}\right]$ (trace) and $\mathrm{PPhTh}_{2}$ (trace), respectively. The third band afforded $\left[\mathrm{Re}_{2}(\mathrm{CO})_{8}\left(\mathrm{PPhTh}_{2}\right)_{2}\right](2)(112 \mathrm{mg}, 66 \%)$ as yellow crystals while the fourth band gave fac[ClRe(CO) $)_{3}\left(\mathrm{PPhTh}_{2}\right)_{2}$ ] (3) (27 mg, 21\%) after recrystallization from hexane $/ \mathrm{CH}_{2} \mathrm{Cl}_{2}$ at $4{ }^{\circ} \mathrm{C}$. Spectral data for 2: Anal. Calc. for $\mathrm{C}_{36} \mathrm{H}_{22} \mathrm{O}_{8} \mathrm{P}_{2} \mathrm{Re}_{2} \mathrm{~S}_{4}: \mathrm{C}, 37.76 ; \mathrm{H}, 1.94$. Found: $\mathrm{C}, 38.03 ; \mathrm{H}, 1.99 \%$. IR $\left(v C O, \mathrm{CH}_{2} \mathrm{Cl}_{2}\right)$ : $2016 \mathrm{w}, 1990 \mathrm{sh}, 1961 \mathrm{vs} \mathrm{cm}^{-1} ;{ }^{1} \mathrm{H}$ NMR $\left(\mathrm{CDCl}_{3}\right): \delta 7.64(\mathrm{~m}, 4 \mathrm{H}), 7.43(\mathrm{~m}, 10 \mathrm{H}), 7.36(\mathrm{~m}, 4 \mathrm{H}), 7.22(\mathrm{~m}$, $4 \mathrm{H}) ;{ }^{31} \mathrm{P}-\left\{{ }^{1} \mathrm{H}\right\}$ NMR $\left(\mathrm{CDCl}_{3}\right): \delta-11.6(\mathrm{~s}) ; \mathrm{MS}(\mathrm{FAB}): \mathrm{m} / z 1146\left(\mathrm{M}^{+}\right)$. Spectral data for 3: Anal. Calc. for $\mathrm{C}_{31} \mathrm{H}_{22} \mathrm{ClO}_{3} \mathrm{P}_{2} \mathrm{ReS}_{4}: \mathrm{C}, 43.58 ; \mathrm{H}, 2.60$. Found: $\mathrm{C}, 43.91 ; \mathrm{H}, 2.66 \%$. IR $\left(v \mathrm{CO}, \mathrm{CH}_{2} \mathrm{Cl}_{2}\right): 2039 \mathrm{~s}, 1960 \mathrm{~m}$, $1907 \mathrm{~m} \mathrm{~cm}^{-1}{ }^{1}{ }^{\mathrm{H}} \mathrm{NMR}\left(\mathrm{CDCl}_{3}\right): \delta 7.56(\mathrm{~m}, 4 \mathrm{H}), 7.49(\mathrm{~m}, 4 \mathrm{H}), 7.33(\mathrm{~m}, 7 \mathrm{H}), 7.23(\mathrm{~m}, 3 \mathrm{H}), 7.03(\mathrm{~m}$, $4 \mathrm{H}) ;{ }^{31} \mathrm{P}-\left\{{ }^{1} \mathrm{H}\right\} \operatorname{NMR}\left(\mathrm{CDCl}_{3}\right): \delta-15.1(\mathrm{~s}) ; \mathrm{MS}(\mathrm{FAB}): \mathrm{m} / \mathrm{z} 854\left(\mathrm{M}^{+}\right)$.

\subsection{Reaction of $[\operatorname{Re} 2(\mathrm{CO}) 10]$ with $\mathrm{PPhTh} 2$}

A xylene solution $(30 \mathrm{~mL})$ of $\left[\operatorname{Re}_{2}(\mathrm{CO})_{10}\right](200 \mathrm{mg}, 0.307 \mathrm{mmol})$ and $\mathrm{PPhTh}(168 \mathrm{mg}, 0.612 \mathrm{mmol})$ was heated to reflux for $7 \mathrm{~h}$. The solvent was removed under reduced pressure and the residue separated by TLC on silica gel. Elution with hexane/ $\mathrm{CH}_{2} \mathrm{Cl}_{2}(7: 3, \mathrm{v} / \mathrm{v})$ developed nine bands. The first and second bands gave unreacted $\left[\mathrm{Re}_{2}(\mathrm{CO})_{10}\right]$ (trace) and $\mathrm{PPhTh}_{2}$ (trace), respectively. The fifth to eighth bands afforded the following compounds in order of elution: $\left[\operatorname{Re}_{2}(\mathrm{CO})_{7}\left(\mathrm{PPhTh}_{2}\right)(\mu-\mathrm{PPhTh})(\mu-\mathrm{H})\right](4)(96 \mathrm{mg}$, $30 \%)$ as colorless crystals, $\left[\operatorname{Re}_{2}(\mathrm{CO})_{7}\left(\mathrm{PPhTh}_{2}\right)(\mu-\mathrm{PPhTh})\left(\mu-\eta^{1}, \mathrm{~K}^{1}(\mathrm{~S})-\mathrm{C}_{4} \mathrm{H}_{3} \mathrm{~S}\right)\right](5)(64 \mathrm{mg}, 19 \%)$ as pale yellow crystals, mer-[HRe(CO) $\left.)_{3}\left(\mathrm{PPhTh}_{2}\right)_{2}\right](6)(43 \mathrm{mg}, 17 \%)$ as colorless crystals and $2(47 \mathrm{mg}, 13 \%)$ after recrystallization from hexane $/ \mathrm{CH}_{2} \mathrm{Cl}_{2}$ at $4{ }^{\circ} \mathrm{C}$. The remaining bands gave minor unidentified products. Spectral data for 4: Anal. Calc. for $\mathrm{C}_{31} \mathrm{H}_{20} \mathrm{O}_{7} \mathrm{P}_{2} \mathrm{Re}_{2} \mathrm{~S}_{3}$ : C, 35.97; $\mathrm{H}, 1.95$. Found: $\mathrm{C}, 36.32 ; \mathrm{H}, 2.03 \%$. IR $\left(v C O, \mathrm{CH}_{2} \mathrm{Cl}_{2}\right.$ ): $2094 \mathrm{~m}, 2048 \mathrm{~m}, 1998 \mathrm{vs}, 1950 \mathrm{~s}, 1924 \mathrm{~s} \mathrm{~cm}^{-1} ;{ }^{1} \mathrm{H} \mathrm{NMR}\left(\mathrm{CDCl}_{3}\right): \delta 7.95(\mathrm{~m}, 2 \mathrm{H}), 7.71(\mathrm{~m}$, $2 \mathrm{H}), 7.53-7.23(\mathrm{~m}, 14 \mathrm{H}), 7.00(\mathrm{~m}, 1 \mathrm{H}),-13.85(\mathrm{dd}, J=16.4,4.8 \mathrm{~Hz}, 1 \mathrm{H}) ;{ }^{31} \mathrm{P}-\left\{{ }^{1} \mathrm{H}\right\} \mathrm{NMR}\left(\mathrm{CDCl}_{3}\right): \delta 24.1$ $(\mathrm{d}, J=70.4 \mathrm{~Hz}, 1 \mathrm{P}),-18.4(\mathrm{~d}, J=70.4 \mathrm{~Hz}, 1 \mathrm{P}) ; \mathrm{MS}(\mathrm{FAB}): \mathrm{m} / z 1034\left(\mathrm{M}^{+}\right)$. Spectral data for 5 : Anal. Calc. for $\mathrm{C}_{35} \mathrm{H}_{22} \mathrm{O}_{7} \mathrm{P}_{2} \mathrm{Re}_{2} \mathrm{~S}_{4}: \mathrm{C}, 37.63 ; \mathrm{H}, 1.98$. Found: $\mathrm{C}, 37.98 ; \mathrm{H}, 2.07 \%$. IR $\left(v \mathrm{CO}, \mathrm{CH}_{2} \mathrm{Cl}_{2}\right): 2082 \mathrm{~m}, 2054 \mathrm{~m}$, 1986 vs, 1974 vs, 1961 vs, $1937 \mathrm{~s} \mathrm{~cm}^{-1}$; ${ }^{1} \mathrm{H}$ NMR $\left(\mathrm{CDCl}_{3}\right): \delta 7.66(\mathrm{~m}, 3 \mathrm{H}), 7.47(\mathrm{~m}, 5 \mathrm{H}), 7.39(\mathrm{~m}, 3 \mathrm{H}), 7.22$ $(\mathrm{m}, 5 \mathrm{H}), 7.06(\mathrm{~m}, 4 \mathrm{H}), 6.90(\mathrm{~m}, 1 \mathrm{H}), 6.38(\mathrm{~m}, 1 \mathrm{H}) ;{ }^{31} \mathrm{P}-\left\{{ }^{1} \mathrm{H}\right\} \mathrm{NMR}\left(\mathrm{CDCl}_{3}\right): \delta-21.0(\mathrm{~d}, \mathrm{~J}=98.0 \mathrm{~Hz}, 1 \mathrm{P})$, $-55.9(\mathrm{~d}, J=98.0 \mathrm{~Hz}, 1 \mathrm{P})$; $\mathrm{MS}(\mathrm{FAB}): \mathrm{m} / z 1118\left(\mathrm{M}^{+}\right)$. Spectral data for 6: Anal. Calc. for $\mathrm{C}_{31} \mathrm{H}_{23} \mathrm{O}_{3} \mathrm{P}_{2} \mathrm{ReS}_{4}$ : $\mathrm{C}, 45.41 ; \mathrm{H}, 2.83$. Found: $\mathrm{C}, 45.73 ; \mathrm{H}, 2.89 \%$. IR ( $\left.v \mathrm{CO}, \mathrm{CH}_{2} \mathrm{Cl}_{2}\right): 1938 \mathrm{vs}, \mathrm{br}, 1917 \mathrm{sh} \mathrm{cm}^{-1} ;{ }^{1} \mathrm{H} \mathrm{NMR}$ $\left(\mathrm{CDCl}_{3}\right): \delta 7.65(\mathrm{~m}, 4 \mathrm{H}), 7.51(\mathrm{~m}, 4 \mathrm{H}), 7.44(\mathrm{~m}, 4 \mathrm{H}), 7.34(\mathrm{~m}, 6 \mathrm{H}), 7.20(\mathrm{~m}, 4 \mathrm{H}),-4.71(\mathrm{t}, J=20.4 \mathrm{~Hz}$, $1 \mathrm{H}) ;{ }^{31} \mathrm{P}-\left\{{ }^{1} \mathrm{H}\right\} \mathrm{NMR}\left(\mathrm{CDCl}_{3}\right): \delta-7.9(\mathrm{~s}) ; \mathrm{MS}(\mathrm{FAB}): \mathrm{m} / \mathrm{z} 820\left(\mathrm{M}^{+}\right)$.

\subsection{Reaction of $[\mathrm{Mn} 2(\mathrm{CO}) 10]$ with $\mathrm{PPhTh} 2$}

A toluene solution $(20 \mathrm{~mL})$ of $\left[\mathrm{Mn}_{2}(\mathrm{CO})_{10}\right](150 \mathrm{mg}, 0.385 \mathrm{mmol})$ and $\mathrm{PPhTh} 2(106 \mathrm{mg}, 0.386 \mathrm{mmol})$ was heated to reflux for $4 \mathrm{~h}$. The solvent was removed under reduced pressure and the residue chromatographed by TLC on silica gel. Elution with hexane/ $\mathrm{CH}_{2} \mathrm{Cl}_{2}(4: 1, \mathrm{v} / \mathrm{v})$ developed five bands. The 
first band gave unreacted [ $\left.\mathrm{Mn}_{2}(\mathrm{CO})_{10}\right]$ (trace). The second, fourth and fifth bands afforded $\left[\mathrm{Mn}_{2}(\mathrm{CO})_{9}\left(\mathrm{PhPTh}_{2}\right)\right](7)(34 \mathrm{mg}, 14 \%)$ as yellow crystals, $\left[\mathrm{Mn}_{2}(\mathrm{CO})_{6}(\mu-\mathrm{PPhTh})\left(\mu-\eta^{1}, \eta^{5}-\mathrm{C}_{4} \mathrm{H}_{3} \mathrm{~S}\right)\right](8)(98 \mathrm{mg}$, $46 \%)$ as orange crystals and $\left[\mathrm{Mn}_{2}(\mathrm{CO})_{5}\left(\mathrm{PPhTh}_{2}\right\}(\mu-\mathrm{PPhTh})\left(\mu-\eta^{1}, \eta^{5}-\mathrm{C}_{4} \mathrm{H}_{3} \mathrm{~S}\right)\right](9)(92 \mathrm{mg}, 30 \%)$ as orange crystals after recrystallization from hexane $/ \mathrm{CH}_{2} \mathrm{Cl}_{2}$ at $4{ }^{\circ} \mathrm{C}$. The third band gave a minor unidentified product. Spectral data for 7: Anal. Calc. for $\mathrm{C}_{23} \mathrm{H}_{11} \mathrm{Mn}_{2} \mathrm{O}_{9} \mathrm{PS}_{2}: \mathrm{C}, 43.41 ; \mathrm{H}, 1.74$. Found: $\mathrm{C}, 43.75 ; \mathrm{H}$, 1.80\%; IR ( $(\mathrm{CO}), \mathrm{CH}_{2} \mathrm{Cl}_{2}$ ): $2091 \mathrm{~m}, 2064 \mathrm{w}, 2012 \mathrm{~s}, 1993 \mathrm{vs}, 1972 \mathrm{~m}, 1958 \mathrm{~m}, 1938 \mathrm{~cm}^{-1}$; ${ }^{1} \mathrm{H}$ NMR $\left(\mathrm{CDCl}_{3}\right): \delta 7.56(\mathrm{~m}, 2 \mathrm{H}), 7.20(\mathrm{~m}, 2 \mathrm{H}), 7.01(\mathrm{~m}, 7 \mathrm{H}) ;{ }^{31} \mathrm{P}-\left\{{ }^{1} \mathrm{H}\right\} \mathrm{NMR}\left(\mathrm{CDCl}_{3}\right): \delta 58.6(\mathrm{~s}) ; \mathrm{MS}(\mathrm{FAB}): \mathrm{m} / z 636$ $\left(\mathrm{M}^{+}\right)$. Spectral data for 8: Anal. Calc. for $\mathrm{C}_{20} \mathrm{H}_{11} \mathrm{Mn}_{2} \mathrm{O}_{6} \mathrm{PS}_{2}$ : $\mathrm{C}, 43.49 ; \mathrm{H}, 2.01$. Found: $\mathrm{C}, 43.81 ; \mathrm{H}, 2.07 \%$; IR

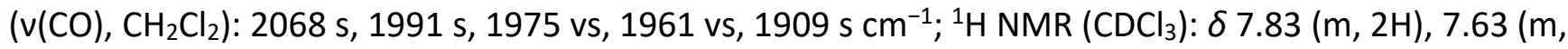
2H), $7.48(\mathrm{~m}, 3 \mathrm{H}), 7.34(\mathrm{~m}, 7 \mathrm{H}), 7.08(\mathrm{~s}, \mathrm{br}, 2 \mathrm{H}), 6.21(\mathrm{~s}, \mathrm{br}, 1 \mathrm{H}), 6.14(\mathrm{~s}, \mathrm{br}, 1 \mathrm{H}), 5.81(\mathrm{~m}, 2 \mathrm{H}), 5.76(\mathrm{~s}, \mathrm{br}$, $1 \mathrm{H}), 5.73(\mathrm{~s}, \mathrm{br}, 1 \mathrm{H}) ;{ }^{1} \mathrm{P}-\left\{{ }^{1} \mathrm{H}\right\}$ NMR $\left(\mathrm{CDCl}_{3}\right): \delta-33.8(\mathrm{~s}, 1 \mathrm{P}),-43.2(\mathrm{~s}, 1 \mathrm{P}) ; \mathrm{MS}(\mathrm{FAB}): \mathrm{m} / z 552\left(\mathrm{M}^{+}\right)$.

Spectral data for 9: Anal. Calc. for $\mathrm{C}_{33} \mathrm{H}_{22} \mathrm{Mn}_{2} \mathrm{O}_{5} \mathrm{P}_{2} \mathrm{~S}_{4}$ : C, 49.63; $\mathrm{H}, 2.78$. Found: $\mathrm{C}, 49.96 ; \mathrm{H}, 2.85 \%$; IR (v(CO), $\left.\mathrm{CH}_{2} \mathrm{Cl}_{2}\right)$ : $2018 \mathrm{w}, 1956 \mathrm{vs}, 1931 \mathrm{vs}, 1911 \mathrm{~s}, 1900 \mathrm{~s} \mathrm{~cm}^{-1} ;{ }^{1} \mathrm{H} \mathrm{NMR}\left(\mathrm{CDCl}_{3}\right): \delta 7.88(\mathrm{~m}, 2 \mathrm{H}), 7.76(\mathrm{~m}$, $2 \mathrm{H}), 7.54(\mathrm{~m}, 4 \mathrm{H}), 7.50-7.27(\mathrm{~m}, 24 \mathrm{H}), 7.12(\mathrm{~m}, 4 \mathrm{H}), 7.04(\mathrm{~m}, 2 \mathrm{H}), 5.85(\mathrm{~s}, \mathrm{br}, 1 \mathrm{H}), 5.75(\mathrm{~s}, \mathrm{br}, 1 \mathrm{H}), 5.66$ $(\mathrm{m}, 2 \mathrm{H}), 5.55(\mathrm{~m}, 1 \mathrm{H}), 5.52(\mathrm{~m}, 1 \mathrm{H}) ;{ }^{31} \mathrm{P}-\left\{{ }^{1} \mathrm{H}\right\} \mathrm{NMR}\left(\mathrm{CDCl}_{3}\right): \delta 51.4(\mathrm{~s}, 2 \mathrm{P}),-12.9(\mathrm{~s}, 1 \mathrm{P}),-21.6(\mathrm{~s}, 1 \mathrm{P}) ; \mathrm{MS}$ (FAB): $m / z 798\left(\mathrm{M}^{+}\right)$.

\subsection{Conversion of 8-9}

A toluene solution $(15 \mathrm{~mL})$ of $8(50 \mathrm{mg}, 0.091 \mathrm{mmol})$ was heated to reflux for $3 \mathrm{~h}$. Workup afforded unreacted 8 (trace) and 9 (39 $\mathrm{mg}, 54 \%)$.

\subsection{X-ray crystallographic studies}

Single crystals of compounds $\mathbf{3}, \mathbf{4}, \mathbf{6 , 8}$ and $\mathbf{9}$ suitable for X-ray diffraction were obtained by recrystallization from hexane $/ \mathrm{CH}_{2} \mathrm{Cl}_{2}$ at room temperature and mounted on Nylon fibers with a mineral oil, and diffraction data were collected at 100(2) K on a Bruker AXS SMART diffractometer equipped with an APEX CCD detector using graphite-monochromated $\mathrm{Cu}$ K $\alpha$ radiation $(\lambda=1.54178 \AA$ ). Integration of intensities and data reduction was performed using the saint program [29]. Numerical (based on the real shape of the crystals) absorption correction was applied in all cases followed by the multiscan sadabs procedure [30]. The structures were solved by direct methods [31] and refined by fullmatrix least-squares on $\mathrm{F}^{2}$ [32]. All non-hydrogen atoms were refined anisotropically. Pertinent crystallographic data and structure refinement parameters are summarized in Table 1. 
Table 1. Crystallographic data for compounds 3, 4, 6, 8 and 9.

\begin{tabular}{|c|c|c|c|c|c|}
\hline Compound & 3 & 4 & 6 & 8 & 9 \\
\hline Empirical formula & $\mathrm{C}_{31} \mathrm{H}_{22} \mathrm{ClO}_{3.30} \mathrm{P}_{2} \mathrm{ReS}_{4}$ & $\mathrm{C}_{31} \mathrm{H}_{20} \mathrm{O}_{7} \mathrm{P}_{2} \mathrm{Re}_{2} \mathrm{~S}_{3}$ & $\mathrm{C}_{31} \mathrm{H}_{23} \mathrm{O}_{3} \mathrm{P}_{2} \mathrm{ReS}_{4}$ & $\mathrm{C}_{20} \mathrm{H}_{11} \mathrm{Mn}_{2} \mathrm{O}_{6} \mathrm{PS}_{2}$ & $\mathrm{C}_{33} \mathrm{H}_{22} \mathrm{Mn}_{2} \mathrm{O}_{5} \mathrm{P}_{2} \mathrm{~S}_{4}$ \\
\hline Formula weight $(\AA ̊)$ & 859.04 & 1034.99 & 819.87 & 552.26 & 798.57 \\
\hline Temperature $(\mathrm{K})$ & $100(2)$ & $100(2)$ & $100(2)$ & $100(2)$ & $100(2)$ \\
\hline Wavelength $(\AA ̊)$ & 1.54178 & 1.54178 & 1.54178 & 1.54178 & 1.54178 \\
\hline Crystal system & monoclinic & monoclinic & monoclinic & monoclinic & monoclinic \\
\hline Space group & $P n$ & $P 2_{1} / n$ & $C 2 / c$ & $P 21 / n$ & $P 2{ }_{1} / c$ \\
\hline \multicolumn{6}{|l|}{ Unit cell dimensions } \\
\hline$a(\AA)$ & $9.5825(3)$ & $13.2604(2)$ & $16.8770(5)$ & $15.9950(4)$ & $18.2626(5)$ \\
\hline$b(\AA)$ & $8.8810(3)$ & $11.4601(2)$ & $9.6013(3)$ & $9.0185(2)$ & $11.8253(3)$ \\
\hline$c(\AA)$ & 18.6992(5) & $22.4574(4)$ & 19.6843(6) & $16.0234(4)$ & $17.0579(5)$ \\
\hline$a\left(^{\circ}\right)$ & 90 & 90 & 90 & 90 & 90 \\
\hline$b\left(^{\circ}\right)$ & $90.5420(10)$ & $104.2630(10)$ & $103.4030(10)$ & $113.2600(10)$ & $117.307(2)$ \\
\hline$c\left(^{\circ}\right)$ & 90 & 90 & 90 & 90 & 90 \\
\hline Volume $\left(\AA^{3}\right)$ & 1591.27(8) & $3307.55(10)$ & $3102.79(16)$ & $2123.53(9)$ & $3273.31(16)$ \\
\hline$Z$ & 2 & 4 & 4 & 4 & 4 \\
\hline $\begin{array}{l}\text { Density (calculated) } \\
\left(\mathrm{Mg} \mathrm{m}^{-3}\right)\end{array}$ & 1.793 & 2.078 & 1.755 & 1.727 & 1.62 \\
\hline $\begin{array}{l}\text { Absorption coefficient } \\
\left(\mathrm{mm}^{-1}\right)\end{array}$ & 11.925 & 17.173 & 11.416 & 12.554 & 9.94 \\
\hline$F\left(\begin{array}{lll}0 & 0 & 0\end{array}\right)$ & 841 & 1960 & 1608 & 1104 & 1616 \\
\hline Crystal size $(\mathrm{mm})$ & $0.37 \times 0.29 \times 0.04$ & $0.42 \times 0.22 \times 0.10$ & $0.23 \times 0.15 \times 0.10$ & $0.49 \times 0.30 \times 0.20$ & $0.40 \times 0.30 \times 0.20$ \\
\hline $\begin{array}{l}\vartheta \text { range for data collection } \\
\left({ }^{\circ}\right)\end{array}$ & $4.73-66.91$ & $4.06-68.02$ & $4.62-67.17$ & $3.31-61.48$ & $2.72-61.32$ \\
\hline \multirow[t]{3}{*}{ Index ranges } & $-11 \leqslant h \leqslant 10$ & $-15 \leqslant h \leqslant 15$ & $-19 \leqslant h \leqslant 19$ & $-18 \leqslant h \leqslant 16$ & $-20 \leqslant h \leqslant 18$ \\
\hline & $-0 \leqslant k \leqslant 10$ & $0 \leqslant k \leqslant 13$ & $0 \leqslant k \leqslant 11$ & $0 \leqslant k \leqslant 10$ & $0 \leqslant k \leqslant 13$ \\
\hline & $-22 \leqslant I \leqslant 21$ & $0 \leqslant 1 \leqslant 26$ & $0 \leqslant 1 \leqslant 22$ & $0 \leqslant I \leqslant 18$ & $0 \leqslant I \leqslant 19$ \\
\hline Reflections collected & 13161 & 27360 & 12995 & 17449 & 26627 \\
\hline $\begin{array}{l}\text { Independent reflections } \\
\left(R_{\text {int }}\right)\end{array}$ & $5170\left[R_{\text {int }}=0.0214\right]$ & $5915\left[R_{\mathrm{int}}=0.0252\right]$ & $2692\left[R_{\text {int }}=0.0177\right]$ & $3225\left[R_{\mathrm{int}}=0.0272\right]$ & $4968\left[R_{\text {int }}=0.0391\right]$ \\
\hline
\end{tabular}




\begin{tabular}{|l|l|l|l|l|l|}
\hline Refinement method & $\begin{array}{l}\text { full-matrix least- } \\
\text { squares on } F^{2}\end{array}$ & $\begin{array}{l}\text { full-matrix least- } \\
\text { squares on } F^{2}\end{array}$ & $\begin{array}{l}\text { full-matrix least- } \\
\text { squares on } F^{2}\end{array}$ & $\begin{array}{l}\text { full-matrix least- } \\
\text { squares on } F^{2}\end{array}$ & $\begin{array}{l}\text { full-matrix least- } \\
\text { squares on } F^{2}\end{array}$ \\
\hline Data/restraints/parameters & $5170 / 301 / 366$ & $5915 / 182 / 389$ & $2692 / 30 / 237$ & $3225 / 30 / 226$ & $4968 / 282 / 382$ \\
\hline Goodness-of-fit (GOF) on $F^{2}$ & 1.003 & 1.039 & 1.11 & 1.203 & 1.019 \\
\hline Final $R$ indices $[I>2 s(I)]$ & $R_{1}=0.0185$, & $R_{1}=0.0307$, & $R_{1}=0.0155$, & $R_{1}=0.0405$, & $R_{1}=0.0621$, \\
\hline & $w R_{2}=0.0457$ & $w R_{2}=0.0736$ & $w R_{2}=0.0391$ & $w R_{2}=0.1003$ & $w R_{2}=0.1474$ \\
\hline$R$ indices (all data) & $R_{1}=0.0189$, & $R_{1}=0.0323$, & $R_{1}=0.0156$, & $R_{1}=0.0414$, & $R_{1}=0.0666$, \\
\hline $\begin{array}{l}\text { Largest diff. peak and hole } \\
\left(\mathrm{e} \AA^{-3}\right)\end{array}$ & $w R_{2}=0.0459$ & $w R_{2}=0.0745$ & $w R_{2}=0.0392$ & $w R_{2}=0.1008$ & $w R_{2}=0.1502$ \\
\hline
\end{tabular}

\section{Results and discussion}

\subsection{Reactions of [Re2(CO)10-n(NCMe)n] ( $n=1,2)$ with PhPTh2: substitution products}

Treatment of $\left[\operatorname{Re}_{2}(\mathrm{CO})_{9}(\mathrm{NCMe})\right]$ with PPhTh 2 at $25^{\circ} \mathrm{C}$ afforded the substitution product $\left[\operatorname{Re}_{2}(\mathrm{CO})_{9}(\mathrm{PPhTh} 2)\right](\mathbf{1})(84 \%)$, while $\left[\operatorname{Re}_{2}(\mathrm{CO})_{8}(\mathrm{NCMe})_{2}\right]$ similarly gave $\left[\operatorname{Re}_{2}(\mathrm{CO})_{8}\left(\mathrm{PPhTh}_{2}\right)_{2}\right](2)(66 \%)$. Both are readily characterized by comparison of spectroscopic data with related $\left[\mathrm{Re}_{2}(\mathrm{CO})_{10-n}(\mathrm{~L})_{n}\right]$ complexes $\left(n=1,2: \mathrm{L}=\mathrm{PPh}_{3}, \mathrm{PMe}_{3}, \mathrm{PTh}{ }_{3}, \mathrm{PFu}\right)$ [27], [28](b), [33]. In the ${ }^{31} \mathrm{P}-\left\{{ }^{1} \mathrm{H}\right\}$ NMR spectra, both display only a singlet, at $\delta-15.6$ for 1 and -11.6 for $\mathbf{2}$, suggesting that the phosphines occupy axial sites in both compounds [27], [33] (Scheme 1).

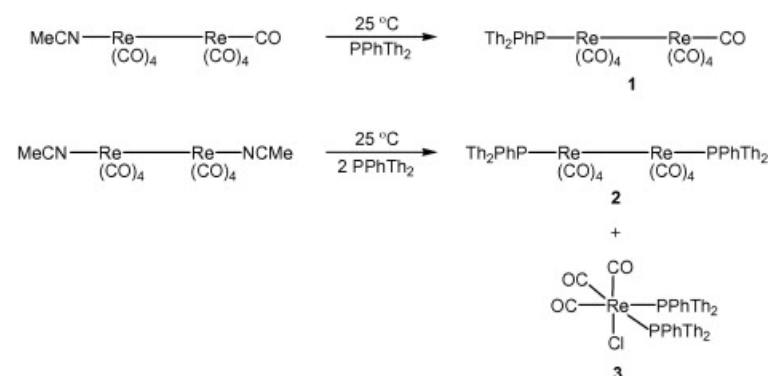

Scheme 1.

An additional product was isolated from the reaction of the bis(acetonitrile) starting material, and it was shown to be fac$\left[\mathrm{ClRe}(\mathrm{CO})_{3}\left(\mathrm{PPhTh}_{2}\right)_{2}\right]$ (3) (21\%) as confirmed by an X-ray crystallographic study. An ORTEP diagram of the molecular structure of $\mathbf{3}$ is depicted in Fig. 1. and selected bond distances and angles are listed in the caption. The basic structure of $\mathbf{3}$ is very similar to that of the recently reported fac-[CIRe(CO) $3\left(\mathrm{~K}^{2}\right.$-dppn)] (dppn = 1,8-bis(diphenylphosphino)naphthalene) [34]. The molecule consists of a single rhenium 
atom with three carbonyl groups arranged in a facial arrangement, two PPhTh 2 ligands and a chloride ligand. The overall coordination geometry is a distorted octahedron which is evident from the expansion of P-Re-P angle to $103.06(2)^{\circ}$, presumably a result of adverse steric interactions. The trans angles about rhenium range from $168.6(3)^{\circ}$ to $176.6(4)^{\circ}$ which are comparable to the corresponding angles in $\mathrm{fac}\left[\mathrm{Re}(\mathrm{CO})_{3}\left(\mathrm{~K}^{1}(\mathrm{P}), \eta^{1}-\mathrm{PPh}_{2} \mathrm{C}_{10} \mathrm{H}_{6}\right)\left(\mathrm{PPh}_{2} \mathrm{H}\right)\right]$ which range from $168.62(9)^{\circ}$ to $172.48(9)^{\circ}$ [34]. The rhenium-phosphorus distances of 2.494(2) $\AA$ and 2.520(2) $\AA$ are similar to those in reported in the literature for related complexes [25], [27], [33], [34]. The source of the chloride ligand was not identified; we speculate that it comes from the chlorinated solvent during chromatographic separation or recrystallization. The rhenium-chlorine bond distance of 2.514(4) $\AA$ is within the expected range [34]. Spectroscopic data for 3 are consistent with the solid-state structure. The ${ }^{1} \mathrm{H}$ NMR spectrum displays only aromatic protons, while the ${ }^{31} \mathrm{P}-\left\{{ }^{1} \mathrm{H}\right\}$ NMR spectrum consists of a singlet at $\delta-15.1$. The FAB mass spectrum shows a parent molecular ion at $m / z 854$.

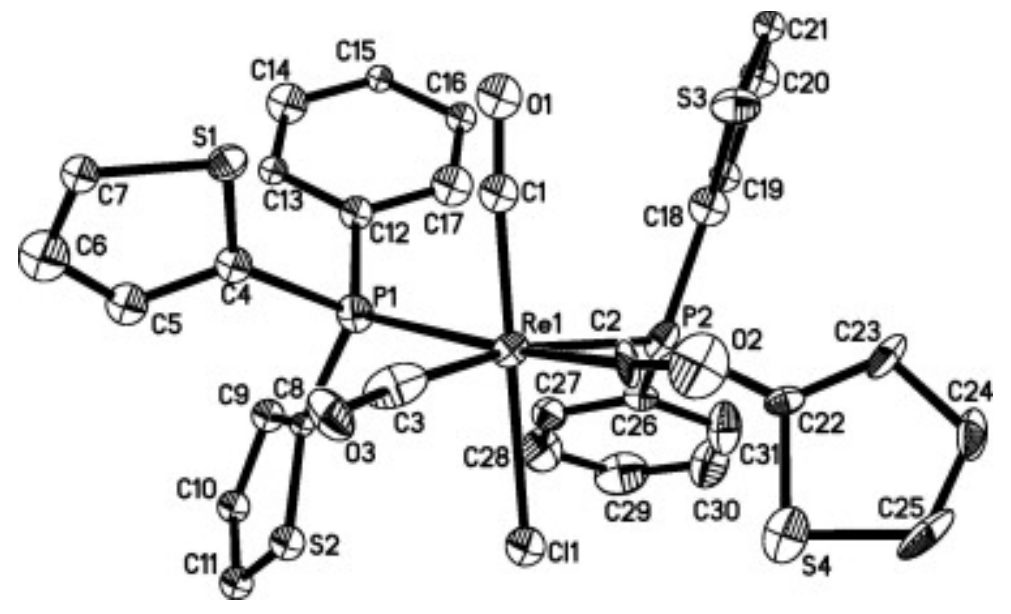

Fig. 1. Molecular structure of fac-[CIRe $\left.(\mathrm{CO})_{3}\left(\mathrm{PPhTh}_{2}\right)_{2}\right](3)$ showing $50 \%$ probability thermal ellipsoids. Ring hydrogens are omitted for clarity. Selected bond lengths $(\AA ̊)$ and angles $\left({ }^{\circ}\right): \operatorname{Re}(1)-P(1) 2.494(2), \operatorname{Re}(1)-P(2) 2.520(2), \operatorname{Re}(1)-C l(1) 2.514(4), \operatorname{Re}(1)-C(1) 1.917(9), \operatorname{Re}(1)-$ $C(2) 1.961(7), \operatorname{Re}(1)-C(3)$ 1.961(10), C(1)-Re(1)-C(2) 89.6(4), C(1)-Re(1)-C(3) 91.2(5), C(2)-Re(1)-C(3) 86.64(12), C(1)-Re(1)-P(1) 82.8(4), $C(1)-\operatorname{Re}(1)-P(2)$ 97.5(4), C(2)-Re(1)-P(1) 168.8(2), C(3)-Re(1)-P(1) 85.3(3), C(2)-Re(1)-P(2) 86.0(3), C(3)-Re(1)-P(2) 168.6(3), C(1)-Re(1)$\mathrm{Cl}(1)$ 176.6(4), C(2)-Re(1)-Cl(1) 92.7(2), C(3)-Re(1)-Cl(1) 91.4(2), P(1)-Re(1)-Cl(1) 95.22(8), Cl(1)-Re(1)-P(2) 80.22(9), P(1)-Re(1)-P(2) 103.06(2), O(1)-C(1)-Re(1) 174.9(12), O(2)-C(2)-Re(1) 175.1(7), O(3)-C(3)-Re(1) 174.3(8). 


\subsection{Thermal reaction of $[\operatorname{Re} 2(\mathrm{CO}) 10]$ and PhPTh2: selective carbon-phosphorus bond scission}

Since at ambient temperature only simple substitution products were observed, we investigated the elevated temperature reaction between $\left[\operatorname{Re}_{2}(\mathrm{CO})_{10}\right]$ and $\mathrm{PPhTh}_{2}$. In refluxing xylene, both $2(13 \%)$ and three new compounds were formed, viz. $\left[\operatorname{Re}_{2}(\mathrm{CO})_{7}(\mathrm{PPhTh})_{2}\right)(\mu-$ $\mathrm{PPhTh})(\mu-\mathrm{H})](4)(30 \%),\left[\operatorname{Re}_{2}(\mathrm{CO})_{7}\left(\mathrm{PPhTh}_{2}\right)(\mu-\mathrm{PPhTh})\left(\mu-\eta^{1}, \mathrm{~K}^{1}(\mathrm{~S})-\mathrm{C}_{4} \mathrm{H}_{3} \mathrm{~S}\right)\right](5)(19 \%)$ and $m e r-\left[\mathrm{He}(\mathrm{CO})_{3}(\mathrm{PPhTh})_{2}\right](6)(17 \%)\left(\mathrm{Scheme}^{2}\right)$.

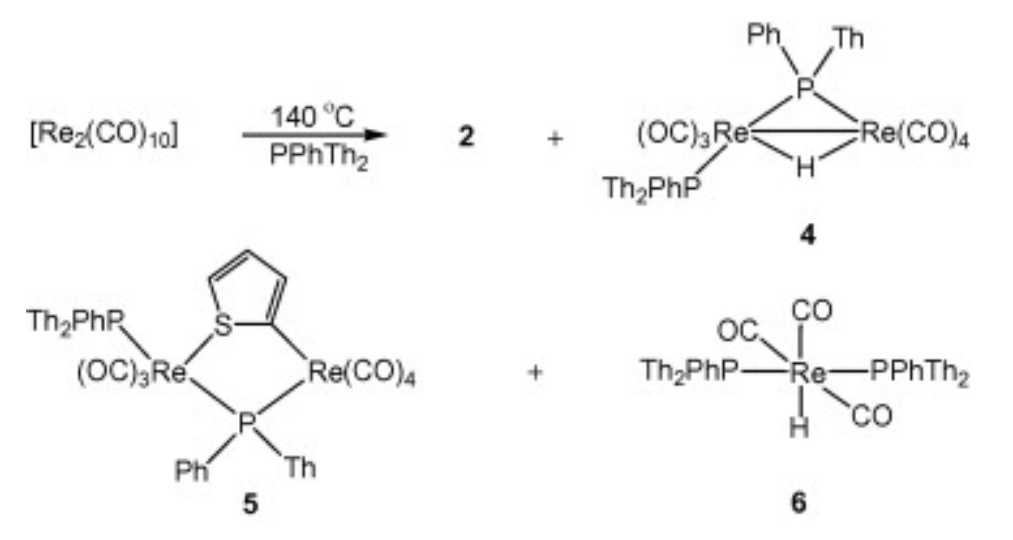

Scheme 2 .

All three new compounds have been characterized by elemental analysis and spectroscopic data, as well as single crystal X-ray diffraction studies for $\mathbf{4}$ and $\mathbf{6}$. An ORTEP diagram of the molecular structure of $\mathbf{4}$ is depicted in Fig. 2. The disordered crystal contains two enantiomers, each containing a dirhenium core with seven terminal carbonyl groups, a bridging phenyl(2-thienyl)phosphido group, an intact $\mathrm{PPhTh}_{2}$ ligand and a bridging hydride. The hydride ligand was located and refined in the structural analysis $[\operatorname{Re}(1)-\mathrm{H}(1 \mathrm{H}) 1.90(7) \AA ̊$ and $\operatorname{Re}(2)-H(1 H) 1.92(7) \AA ̊]$. The phenyl(2-thienyl)phosphido ligand bridges the rhenium-rhenium vector somewhat asymmetrically [Re(1)- $P(1)$

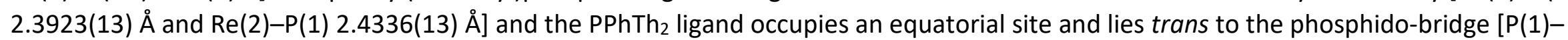

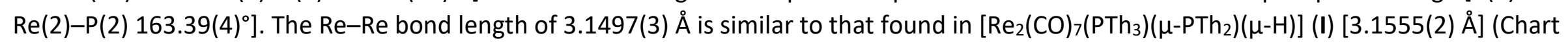
2) and the IR spectrum also closely resembles that of I [33]. In the solid-state structure the thienyl and phenyl groups are clearly distinguished, but occupy chemically inequivalent sites. The ${ }^{31} \mathrm{P}-\left\{{ }^{1} \mathrm{H}\right\}$ NMR spectrum clearly indicates the existence of two isomeric forms in solution in an approximate 10:1 ratio. The major isomer (probably that seen in the solid-state (4a, Chart 3), with the phosphine in relative trans position to the thienyl substituent of the bridging phosphido moiety), consists of two doublets at $\delta 24.1$ and -18.4 $\left(J_{P P}=70.4 \mathrm{~Hz}\right)$, while there is also a second set of doublets at $\delta-8.4$ and $-17.9\left(J_{P P}=76.4 \mathrm{~Hz}\right)$ attributed to the minor isomer $($ Chart 3$)$. Both sets of signals are sharp and we have no evidence for the interconversion of the two. Apparently, this isomer has the phosphine in 
the cis position relative to the bridging phosphido moiety. Steric crowding of the P-P groupings gives energies of $861 \mathrm{vs.} 843 \mathrm{~kJ} / \mathrm{mol}$ for the trans isomer. The crystal of 4 a contains two enantiomers in a 50/50 ratio (Chart 3 ).

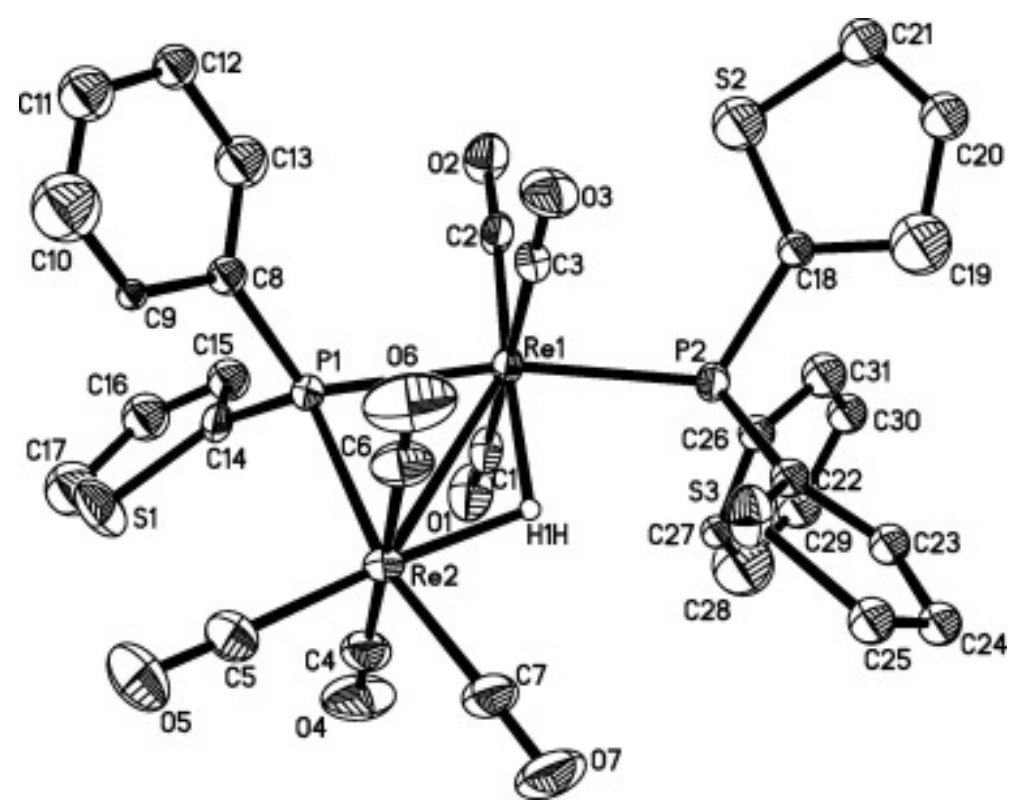

Fig. 2. Molecular structure of $\left[\operatorname{Re}_{2}(\mathrm{CO})_{7}(\mathrm{PPhTh})(\mu-\mathrm{PPhTh})(\mu-\mathrm{H})\right](4)$ showing $50 \%$ probability thermal ellipsoids. Ring hydrogens are omitted for clarity. Selected bond lengths ( $\AA$ ) and angles ( $\left.{ }^{\circ}\right)$ : $\operatorname{Re}(1)-\operatorname{Re}(2) 3.1497(3), \operatorname{Re}(1)-P(1) 2.3923(13), \operatorname{Re}(1)-P(2) 2.4162(12), \operatorname{Re}(2)-P(1)$ 2.4336(13), Re(1)-H(1H) 1.90(7), Re(2)-H(1H) 1.92(7), C(2)-Re(1)-C(3) 88.8(2), C(2)-Re(1)-C(1) 87.6(2), C(3)-Re(1)-C(1) 176.4(2), C(2)$\operatorname{Re}(1)-P(1)$ 100.08(15), $C(3)-\operatorname{Re}(1)-P(1)$ 91.09(15), C(1)-re(1)-P(1) 90.17(16), C(2)-Re(1)-P(2) 96.31(15), C(3)-Re(1)-P(2) 86.56(15), C(1)$\operatorname{Re}(1)-P(2)$ 93.19(15), P(1)-Re(1)-P(2) 163.39(4), P(1)-Re(1)-Re(2) 49.83(3), P(1)-Re(2)-Re(1) 48.69(3), P(1)-Re(1)-H(1H) 84(2), P(1)-Re(2)$\mathrm{H}(1 \mathrm{H})$ 83(2), P(2)-Re(1)-H(1H) 80(2), C(7)-Re(2)-H(1H) 83(2), C(2)-Re(1)-H(1H) 174(2), C(5)-Re(2)-H(1H) 176(2), Re(2)-Re(1)-H(1H) 35(2), $\operatorname{Re}(1)-\operatorname{Re}(2)-H(1 H) 34(2), C(7)-\operatorname{Re}(2)-P(1)$ 165.63(19), C(6)-Re(2)-P(1) 89.35(17), C(5)-Re(2)-P(1) 100.5(2), C(5)-Re(2)-C(7) 93.8(3), C(5)$\operatorname{Re}(2)-C(6)$ 89.1(3), C(5)-Re(2)-C(4) 91.5(3), C(6)-Re(2)-C(4) 178.1(2), Re(1)-P(1)-Re(2) 81.48(4), C(14)-P(1)-C(8) 104.6(5). 


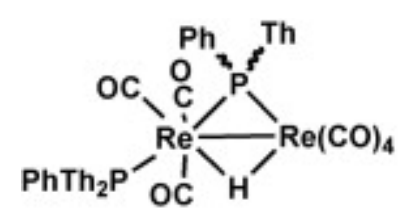

$4 a$

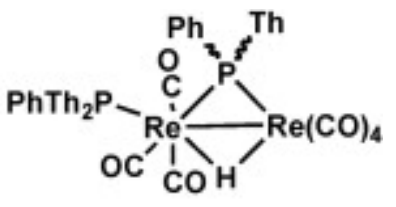

4b

Chart 3.

All attempts to obtain single crystals of $\mathbf{5}$ suitable for X-ray diffraction analysis were unsuccessful and characterization has therefore been made on the basis of analytical and spectroscopic data. The IR spectrum is very similar to that of $\left[\operatorname{Re}_{2}(C)_{7}\left(\mathrm{PTh}_{3}\right)\left(\mu-\mathrm{PTh}_{2}\right)\left(\mu-\eta^{1}, \mathrm{~K}^{1}(\mathrm{~S})-\mathrm{C}_{4} \mathrm{H}_{3} \mathrm{~S}\right)\right]$ (J) (Chart 2), indicating that the two are isostructural. The ${ }^{1} \mathrm{H}$ NMR spectrum shows seven multiplets in the aromatic region with the relative intensity of 3:5:3:5:4:1:1 which are assigned to the phenyl and thienyl ring protons. In addition, the multiplet at $\delta 6.38$ (integrating to $1 \mathrm{H}$ ) is indicative of an orthometallated thienyl or phenyl ring. The ${ }^{31} \mathrm{P}-\left\{{ }^{1} \mathrm{H}\right\}$ NMR spectrum again indicates the presence of two isomers in solution in an approximate 20:1 ratio. The major isomer shows doublets at $\delta-21.0$ and $-55.9\left(J_{P P}=98.0 \mathrm{~Hz}\right)$, while the minor exhibits doublets at $\delta-19.1(J=116.4 \mathrm{~Hz})$ and $-53.7\left(J_{P P}=116.4 \mathrm{~Hz}\right)$; these isomers are probably isomers (diastereomers) of the same kind observed for 4 , but may also be diastereomers formed by the combination of the stereogenic centers at the sulfur and the bridging phosphido moiety (or combinations of these types of isomers). The FAB mass spectrum shows a molecular ion at $\mathrm{m} / \mathrm{z} 1118$ and fragmentation ions due to sequential loss of seven carbonyl groups. Elemental analysis (cf. Section 2.3) was also consistent with the empirical formula.

An ORTEP diagram of the molecular structure of 6 is shown in Fig. 3. and selected bond distances and angles are listed in the caption. The molecule has two crystallographic planes of symmetry and contains a single rhenium atom ligated by three carbonyls, two phosphines and a hydride. The latter was located and refined in the structural analysis $[\operatorname{Re}(1)-H(1 H) 1.73(6) \AA]$. The PPhTh2 ligands lie trans $[P(1)-\operatorname{Re}(1)-$ $\mathrm{P}(1) \# 1$ 166.47(3) $)^{\circ}$ presumably to minimize steric hindrance. While in $\mathbf{3}$ the carbonyls are arranged in a facial fashion in 6 they adopt a meridional arrangement. The structure is very similar to that of mer-[HRe(CO) $\left.)_{3}\left(\mathrm{PPh}_{3}\right)_{2}\right]$ [35] and mer-[HRe(CO) $\left.)_{3}(\mathrm{PTh})_{2}\right)_{2}[27]$. Spectroscopic data suggests that the solid-state structure persists in solution. The ${ }^{1} \mathrm{H}$ NMR spectrum shows a triplet at $\delta-4.71\left(J_{P H}=20.4 \mathrm{~Hz}\right)$ due to the hydride, while the ${ }^{31} \mathrm{P}\left\{{ }^{1} \mathrm{H}\right\}$ NMR spectrum contains only a singlet at $\delta-7.9$ due to two equivalent phosphorus atoms. 


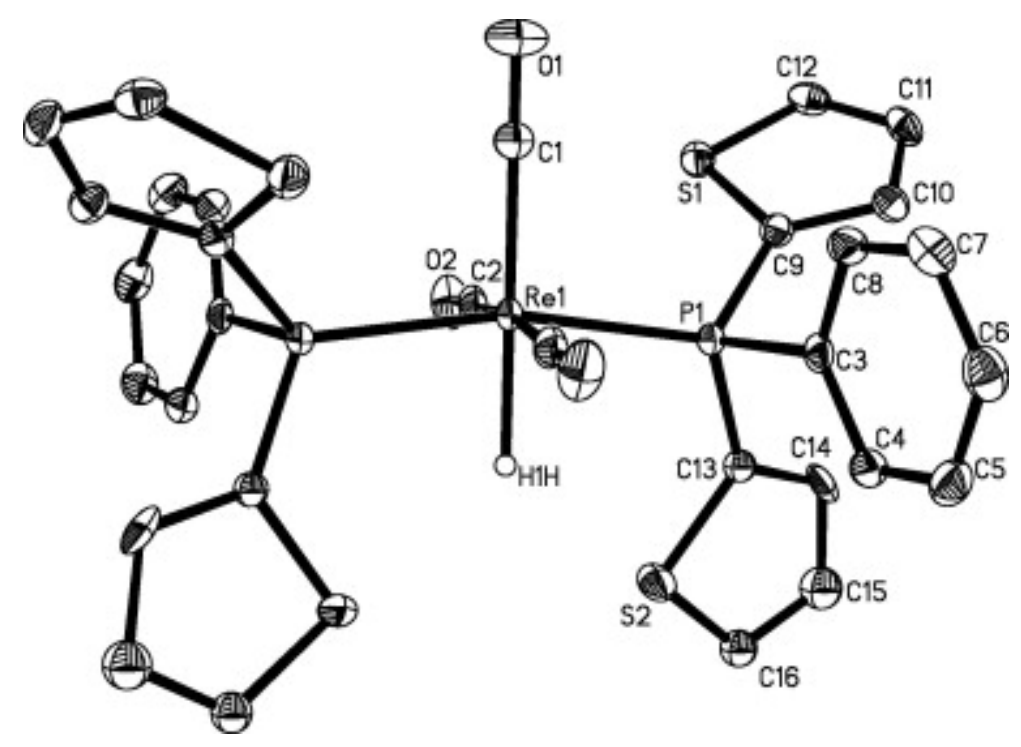

Fig. 3. Molecular structure of $\operatorname{mer}-\left[\mathrm{HRe}(\mathrm{CO})_{3}\left(\mathrm{PPhTh}_{2}\right)_{2}\right](6)$ showing $50 \%$ probability thermal ellipsoids. Ring hydrogens are omitted for clarity. Selected bond lengths $(\AA)$ and angles $\left(^{\circ}\right)$ : $\operatorname{Re}(1)-P(1) 2.3849(5), \operatorname{Re}(1)-P(1) \# 12.3849(5), \operatorname{Re}(1)-H(1 H) 1.73(6), \operatorname{Re}(1)-C(1) 1.957(3)$, $\operatorname{Re}(1)-C(2) 1.986(2), \operatorname{Re}(1)-C(2) \# 1$ 1.986(2), C(1)-Re(1)-C(2) 93.41(6), C(1)-Re(1)-C(2)\#1 93.41(6), C(2)-Re(1)-C(2)\#1 173.18(12), C(1)$\operatorname{Re}(1)-P(1)$ 96.767(13), C(1)-Re(1)-P(1)\#1 96.766(12), C(2)-Re(1)-P(1) 91.11(6), C(2)\#1-Re(1)-P(1) 88.09(6), C(2)-Re(1)-P(1)\#1 88.09(6), $\mathrm{C}(2) \# 1-\operatorname{Re}(1)-\mathrm{P}(1) \# 1$ 91.10(6), C(1)-Re(1)-H(1H) 180.00(2), C(2)-Re(1)-H(1H) 86.59(6), C(2)\#1-Re(1)-H(1H) 86.59(6), P(1)-Re(1)-H(1H) 83.233(13), P(1)\#1-Re(1)-H(1H) 83.23(2), P(1)-Re(1)-P(1)\#1 166.47(3), O(1)-C(1)-Re(1) 180.000(1), O(2)-C(2)-Re(1) 177.24(19).

\subsection{Reaction of [Mn2(CO)10] with PPhTh2: carbon-phosphorus bonds cleavage}

Since the reactivity of $\left[\mathrm{Mn}_{2}(\mathrm{CO})_{10}\right.$ ] with $\mathrm{PPh}_{2} \mathrm{Th}$ [25] closely parallels that established previously for $\mathrm{PTh}_{3}$ [27], we also conducted for comparison the reaction of $\left[\mathrm{Mn}_{2}(\mathrm{CO})_{10}\right]$ with $\mathrm{PPhTh}_{2}$. In refluxing toluene three new complexes were formed, namely $\left[\mathrm{Mn}_{2}(\mathrm{CO})_{9}(\mathrm{PPhTh})_{2}\right)$ (7) (14\%), $\left[\mathrm{Mn}_{2}(\mathrm{CO})_{6}(\mu-\mathrm{PPhTh})\left(\mu-\eta^{1}, \eta^{5}-\mathrm{C}_{4} \mathrm{H}_{3} \mathrm{~S}\right)\right](8)(46 \%)$ and $\left.\left[\mathrm{Mn}_{2}(\mathrm{CO})_{5}(\mathrm{PPhTh})_{2}\right)(\mu-\mathrm{PPhTh})\left(\mu-\eta^{1}, \eta^{5}-\mathrm{C}_{4} \mathrm{H}_{3} \mathrm{~S}\right)\right]$ (9) (30\%) (Scheme 3). In a separate experiment we also showed that $\mathbf{8}$ is converted into 9 when treated with $\mathrm{PPhTh}_{2}$ under the same conditions. These results are similar to those observed for $\mathrm{PTh}_{3}$ [27]. The structure of $\mathbf{7}$ is easily assigned on the basis of spectroscopic data - the IR spectrum being very similar to other substituted [Mn(CO) $\left.{ }_{9} \mathrm{~L}\right]$ compounds ( $\mathrm{L}=\mathrm{PTh} 3, \mathrm{PPhTh}_{2}$, diphenyl(1-naphthyl)phosphine) [25], [27], [34]. The ${ }^{31} \mathrm{P}-\left\{{ }^{1} \mathrm{H}\right\}$ NMR spectrum displays only a singlet at $\delta 58.6$ and the FAB mass spectrum exhibits a parent molecular ion at $\mathrm{m} / \mathrm{z} 636 \mathrm{consistent}$ with the proposed structure. 

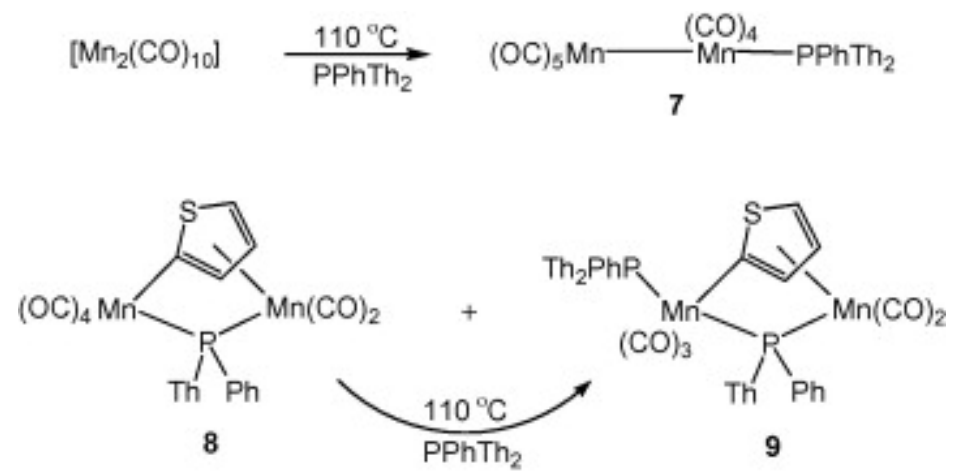

Scheme 3.

An ORTEP diagram of the molecular structure of $\mathbf{8}$ is shown in Fig. 4. and selected bond distances and angles are listed in the caption. The molecule consists of a dinuclear framework of two manganese atoms coordinated by six carbonyl groups, a phenylthienyl phosphido bridge and a thienyl bridge. The structure is very similar to the $\mathrm{PPh}_{2}$ Th and $\mathrm{PTh}_{3}$ analogs $\left[\mathrm{Mn}_{2}(\mathrm{CO})_{6}\left(\mu-\mathrm{PPh}_{2}\right)\left(\mu-\eta^{1}, \eta^{5}-\mathrm{C}_{4} \mathrm{H}_{3} \mathrm{~S}\right)\right](\mathrm{D})(\mathrm{Chart} 1)$ and $\left.\left[\mathrm{Mn}_{2}(\mathrm{CO})_{6}(\mu-\mathrm{PTh})_{2}\right)\left(\mu-\eta^{1}, \eta^{5}-\mathrm{C}_{4} \mathrm{H}_{3} \mathrm{~S}\right)\right](\mathrm{O})$ (Chart 2). The phosphido ligand bridges the dimanganese center [Mn...Mn 3.510(1) $\AA$ ] quite asymmetrically [Mn(1)-P(1) 2.3711(11) $\AA$ and $M n(2)-P(1) 2.2848(11) \AA]$. The bridging thienyl ligand is bound to one manganese atom by a simple $\sigma$-bond $[\mathrm{Mn}(1)-\mathrm{C}(10) 2.079(4) \AA]$, but it binds to the second in an $\eta^{5}$-fashion with manganese-carbon bond distances ranging between 2.129(4) and 2.195(4) $\AA$. This is very similar to the situation found in $\mathbf{D}$ and $\mathbf{O}$ and we therefore conclude that it acts as an overall 7electron donor ligand. As a result, $\mathrm{Mn}(1)$ has an octahedral coordination sphere, while $\mathrm{Mn}(2)$ possesses a half-sandwich geometry. Both ${ }^{1} \mathrm{H}$ NMR and ${ }^{31} \mathrm{P}-\left\{{ }^{1} \mathrm{H}\right\}$ NMR spectra suggest that it exists in two isomeric (diastereomeric) forms in solution. For example, the latter spectrum shows two equal intensity singlets at $\delta-33.8$ and -43.2 . As discussed above, it is likely that the diastereomers are formed by the combination of the stereogenic centers at the sulfur and the bridging phosphido moiety (or combinations of these types of isomers). The $\pi$ complexation of a thienyl ring is also indicated by the upfield resonances in the ${ }^{1} \mathrm{H}$ NMR spectrum at $\delta 6.21,6.14,5.81,5.76$ and 5.73 in a 1:1:2:1:1 ratio. The FAB mass spectrum exhibits a parent molecular ion at $m / z 552$ plus other ions due to stepwise loss of six carbonyl ligands, which is in accord with the solid-state structue. 


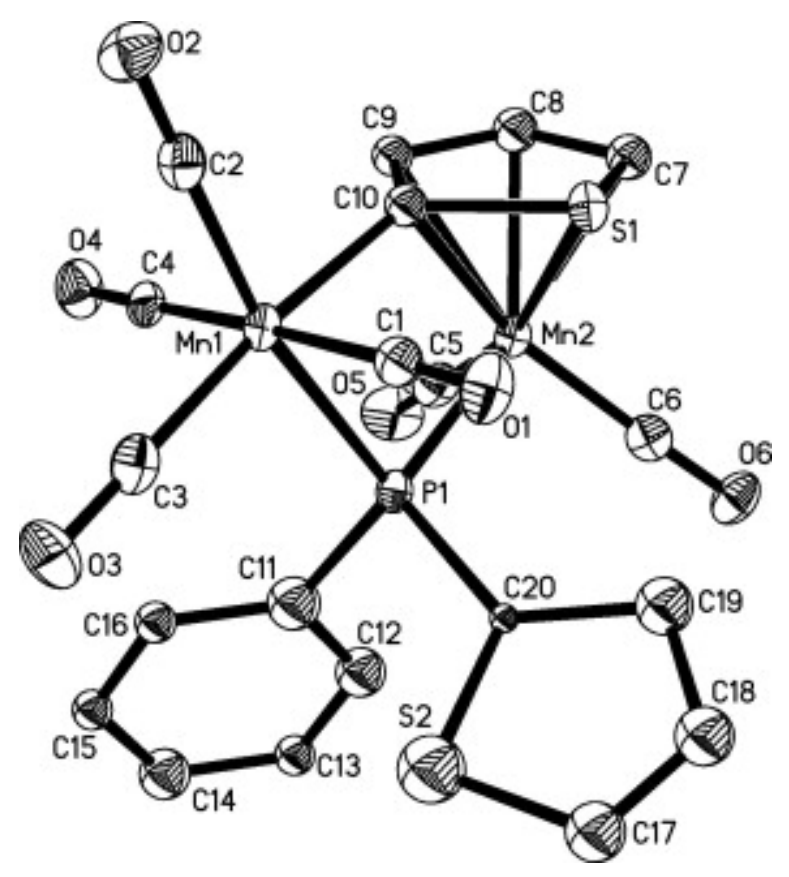

Fig. 4. Molecular structure of $\left[\mathrm{Mn}_{2}(\mathrm{CO})_{6}(\mu-\mathrm{PPhTh})\left(\mu-\eta^{1}, \eta^{5}-\mathrm{C}_{4} \mathrm{H}_{3} \mathrm{~S}\right)\right](8)$ showing $50 \%$ probability thermal ellipsoids. Ring hydrogens are omitted for clarity. Selected bond lengths ( $(\AA)$ and angles ( $\left.{ }^{\circ}\right)$ : $M n(1)-P(1)$ 2.3711(11), $M n(2)-P(1) 2.2848(11), M n(2)-S(1) 2.3084(10), M n(2)-$ $C(7)$ 2.129(4), $M n(2)-C(8)$ 2.156(4), $M n(2)-C(9)$ 2.177(4), $M n(2)-C(10)$ 2.195(4), $M n(1)-C(10)$ 2.079(4), C(3)-Mn(1)-C(2) 95.35(17), C(1)$\mathrm{Mn}(1)-\mathrm{C}(4)$ 176.33(16), C(3)-Mn(1)-C(10) 171.62(16), C(2)-Mn(1)-C(10) 92.85(16), C(3)-Mn(1)-P(1) 96.13(13), C(2)-Mn(1)-P(1) 168.29(12), C(10)-Mn(1)-P(1) 75.76(10), C(1)-Mn(1)-P(1) 86.82(12), C(1)-Mn(1)-C(10) 92.89(15), C(5)-Mn(2)-P(1) 93.65(13), C(6)-Mn(2)$\mathrm{P}(1)$ 99.04(12), C(5)-Mn(2)-C(6) 88.73(18), C(10)-Mn(2)-P(1) 75.44(10), P(1)-Mn(2)-S(1) 92.31(4), Mn(2)-P(1)-Mn(1) 97.86(4), Mn(1)$\mathrm{C}(10)-\mathrm{Mn}(2) 110.40(16), \mathrm{C}(20)-\mathrm{P}(1)-\mathrm{C}(11) 101.1(4)$.

An ORTEP diagram of the molecular structure of 9 is shown in Fig. 5. and selected bond distances and angles are listed in the caption. The structure is related to that of $\mathbf{8}$ in with a PPhTh 2 ligand replacing an equatorial $\mathrm{CO}$ group on $\mathrm{Mn}(1)$ that lies trans to the phosphido bridge. This structure is also very similar to that of $\left[\mathrm{Mn}_{2}(\mathrm{CO})_{5}\left(\mathrm{PTh}_{3}\right)(\mu-\mathrm{PTh})\left(\mu-\eta^{1}, \eta^{5}-\mathrm{C}_{4} \mathrm{H}_{3} \mathrm{~S}\right)\right](\mathbf{9})(\mathbf{P})$ (Chart 2) This complex is disordered in the crystal so that the two diastereomers overlap in a 50/50 ratio. In addition the crystal is centric so that there is an exact 50/50 ratio of the enantiomeric forms of each diastereomer. The ${ }^{31} \mathrm{P}-\left\{{ }^{1} \mathrm{H}\right\}$ NMR spectrum displays three resonances at $\delta 51.4,-12.9$ and -21.6 in a 2:1:1 ratio. The FAB mass spectrum exhibits the parent molecular ion at $m / z 798$ together with ions due to successive loss of five carbonyl ligands which is consistent with the solid-state structue. 


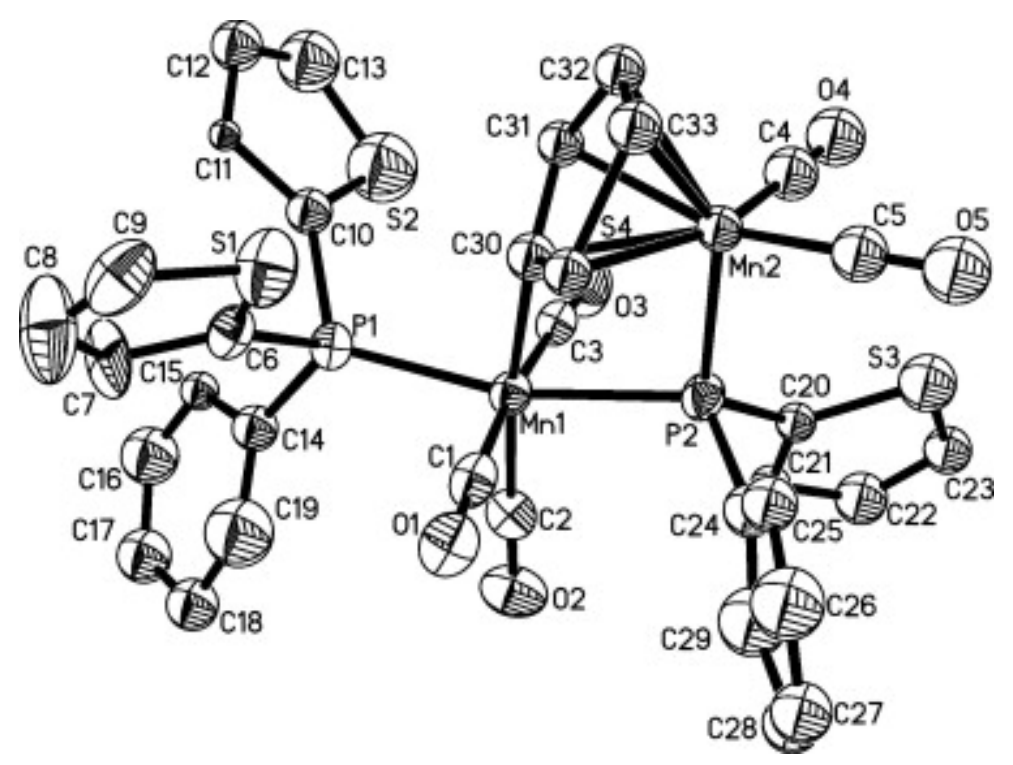

Fig. 5. Molecular structure of $\left[\mathrm{Mn}_{2}(\mathrm{CO})_{5}\left(\mathrm{PPhTh}_{2}\right)(\mu-\mathrm{PPhTh})\left(\mu-\eta^{1}, \eta^{5}-\mathrm{C}_{4} \mathrm{H}_{3} \mathrm{~S}\right)\right](9)$ showing $50 \%$ probability thermal ellipsoids. Ring hydrogens are omitted for clarity. Selected bond lengths $(\AA ̊)$ and angles $\left({ }^{\circ}\right): M n(1)-P(1) 2.2880(15), M n(1)-P(2) 2.3471(15), M n(2)-P(2) 2.241(2)$, $M n(2)-S(4)$ 2.295(4), $M n(1)-C(30)$ 2.080(10), $M n(2)-C(30)$ 2.198(12), $M n(2)-C(31)$ 2.186(11), $M n(2)-C(32) 2.161(11), M n(2)-C(33)$ 2.119(11), C(1)-Mn(1)-C(3) 174.2(2), C(2)-Mn(1)-C(30) 169.5(4), C(2)-Mn(1)-P(1) 97.98(18), C(30)-Mn(1)-P(1) 90.7(4), C(30)-Mn(1)-P(2) 75.9(4), P(1)-Mn(1)-P(2) 165.69(6), C(1)-Mn(1)-P(2) 92.02(16), C(1)-Mn(1)-P(1) 92.03(16), C(1)-Mn(1)-C(30) 86.0(4), C(4)-Mn(2)-C(5) 89.3(7), C(4)-Mn(2)-P(2) 91.6(4), C(5)-Mn(2)-P(2) 97.3(4), P(2)-Mn(2)-S(4) 92.57(10), Mn(2)-P(2)-Mn(1) 98.28(7), Mn(1)-C(30)-Mn(2) 108.4(6), C(24)-P(2)-C(20) 95.8(9).

\section{Conclusion}

In summary, we have demonstrated the reactivity of $\operatorname{PPhTh}_{2}$ towards $\left[\operatorname{Re}_{2}(\mathrm{CO})_{10-x}(\mathrm{NCMe})_{x}\right](x=0,1,2)$ and $\left[\mathrm{Mn} 2(\mathrm{CO})_{10}\right]$. Under mild conditions, reactions with the lightly stabilized rhenium complexes give simple substitution products, but carbon-phosphorus bond cleavage is observed under more forcing conditions. Reaction of $\left[\mathrm{Mn}_{2}(\mathrm{CO})_{10}\right]$ with $\mathrm{PPhTh}_{2}$ affords three dimanganese complexes of which compounds 8 and 9 have been obtained as a result of carbon-phosphorus bond cleavage and each contains a bridging thienyl ligand bonded to one manganese atom in a $\eta^{5}$-fashion. This observation is similar to that reported for $\mathrm{PPh}_{2} \mathrm{Th}_{\text {[25] }}$ and PTh [27]. We note that in all instances it is the phosphorus-thienyl bond that is cleaved. 


\section{Supplementary material}

CCDC $667828,694818,667827,634409$ and 634410 contain the supplementary crystallographic data for 3, 4, 6, 8 and $\mathbf{9}$, respectively. These data can be obtained free of charge from The Cambridge Crystallographic Data Centre via www.ccdc.cam.ac.uk/data_request/cif.

\section{Acknowledgements}

N.B. thanks Sher-e-Bangla Agricultural University for leave to work at Lund University. This research has been sponsored by the Swedish Research Council (VR), the Royal Swedish Academy of Sciences, the Swedish International Development Agency (SIDA) and Ministry of Science and Information \& Communication Technology, Government of the People's Republic of Bangladesh.

\section{References}

[1] H. Topsøe, B.A. Clausen, F.E. Massoth. Hydrotreating Catalysis. Springer, Berlin (1996)

[2] J.G. Speight (Ed.), Petroleum Chemistry and Refining, Taylor and Francis, Washington, DC (1998)

[3] R.J. Angelici. R.B. King (Ed.), Encyclopedia of Inorganic Chemistry, vol. 3, Wiley, New York (1994), pp. 1433-1443

[4] (a) D.D. Whitehurst, T. Isoda, I. Mochida. Adv. Catal., 42 (1998), p. 345 (b) M.J. Ledoux, O. Michaux, G. Agostini, P. Panissod. J. Catal., 102 (1986), p. 275

[5]_(a) B.C. Gates, J.R. Katzer, G.C.A. Schuit. Chemistry of Catalytic Processes. McGraw-Hill, New York (1979). pp. 390-447. (b) G.D. Galpern, in: S. Gronowitz (Ed.), The Chemistry of Heterocyclic Compounds, vol. 44, Part 1, Wiley, New York, 1985, pp. 325-351.

[6]_(a) R.A. Sanchez-Delgado. J. Mol. Catal., 86 (1994), p. 287. (b) R.J. Angelici. Polyhedron, 16 (1997), p. 3073. (C) C. Bianchini, A. Meli. J. Chem. Soc., Dalton Trans. (1996), p. 801

[7]_(a) R.D. Adams, O.-S. Kwon, J.L. Perrin. J. Organomet. Chem., 596 (2000), p. 102. (b) R.D. Adams, X. Qu. Organometallics, 14 (1995), p. 2238

[8]_(a) T.A. Pecoraro, R.R. Chianelli. J. Catal., 67 (1981), p. 430. (b) R.R. Chianelli. Catal. Rev., 26 (1984), p. 361. (c)C. Bianchini, A. Meli. Acc. Chem. Res., 31 (1998), p. 109. (d) T.B. Rauchfuss. Prog. Inorg. Chem., 39 (1991), p. 259. (e) D.A. Vicic, W.D. Jones. J. Am. Chem. Soc., 121 (1999), p. 7606

[9]_(a) R.J. Angelici. Acc. Chem. Res., 21 (1988), p. 387. (b) N.N. Sauer, R.J. Angelici. J. Catal., 116 (1989), p. 11.

(c)J.J. Garcia, B.E. Mann, H. Adams, N.A. Bailey, P.M. Maitlis. J. Am. Chem. Soc., 117 (1995), p. 2179. (d) R.J. Angelici.

Organometallics, 20 (2001), p. 1259. and references therein

[10]_(a) R.J. Angelici. Coord. Chem. Rev., 105 (1990), p. 61. (b) R.J. Angelici. Coord. Chem. Rev., 206-207 (2000), p. 63

[11] M. Brorson, J.D. King, K. Kiriakidou, F. Prestopino, E. Nordlander. P. Braunstein, L.A. Oro, P.R. Raithby (Eds.), Metal Clusters in Chemistry, vol. 2, Wiley-VCH, Weinheim (1999), pp. 741-781. Chapter 2.6 
[12]_(a) R.J. Angelici, Transition Metal Sulphides, NATO ASI Ser 3, vol. 60, 1998, p. 89. (b) C. Bianchini, A. Meli, Transition Metal Sulphides, NATO ASI Ser. 3, vol. 60, 1998, p. 129. (c)R.J. Angelici. Bull. Soc. Chim. Belg., 104 (1995), p. 265

[13]_(a) P.A. Vecchi, A. Ellern, R.J. Anjelici. Organometallics, 24 (2005), p. 2168. (b) P.A. Vecchi, A. Ellern, R.J. Anjelici. J. Am. Chem. Soc., 125 (2003), p. 2064

[14]_(a) H. Li, K. Yu, E.J. Watson, K.L. Virkaitis, J.S. D’Acchioli, G.B. Carpenter, D.A. Sweigart. Organometallics, 21 (2002), p. 1262. (b) A.J. Hernández-Maldonado, R.T. Yang. J. Am. Chem. Soc., 126 (2004), p. 992

[15]_(a) M.A. Reynolds, I.A. Guzei, R.J. Angelici. J. Am. Chem. Soc., 124 (2002), p. 1689. (b) M.A. Reynolds, I.A. Guzei, R.J. Angelici. Organometallics, 20 (2001), p. 1071

[16]_(a) D.G. Churchill, B.M. Bridgewater, G. Parkin. J. Am. Chem. Soc., 122 (2000), p. 178. (b) M.S. Palmer, S. Harris. Organometallics, 19 (2000), p. 2114. (c)J. Torres-Nieto, A. Arévalo, P. García-Gutiérrez, A. Acosta-Ramírez, J.J. García. Organometallics, 23 (2004), p. 4534

[17]_(a) K. Bieger, F. Estevan, P. Lahuerta, J. Lloret, J. Pérez-Prieto, M. Sanaú, N. Siguero, S.-E. Stiriba. Organometallics, 22 (2003), p. 1799. (b) J. Lloret, F. Estevan, P. Lahuerta, P. Hirva, J. Pérez-Prieto, M. Sanaú. Organometallics, 25 (2006), p. 3156

[18] A.J. Deeming, S.N. Jayasuriya, A.J. Arce, Y.D. Sanctis. Organometallics, 15 (1996), p. 786.

[19] J.D. King, M. Monari, E. Nordlander. J. Organomet. Chem., 573 (1999), p. 272

[20] N.K. Kiriakidou Kazemifar, M.J. Stchedroff, M.A. Mottalib, S. Selva, M. Monari, E. Nordlander. Eur. J. Inorg. Chem. (2006), p. 2058

[21] S.P. Tunik, I.G. Koshevoy, A.J. Poë, D.H. Farrar, E. Nordlander, M. Haukka, P.A. Pakkanen. J. Chem. Soc., Dalton Trans. (2003), p. 2457

[22] N.K. Kiriakidou Kazemifar, M.J. Stchedroff, M.H. Johannson, M.A. Mottalib, M. Monari, E. Nordlander, Unpublished results.

[23] M.A. Mottalib, S.E. Kabir, D.A. Tocher, A.J. Deeming, E. Nordlander. J. Organomet. Chem., 692 (2007), p. 5007

[24] U. Bodensieck, H. Varenkamp, G. Rheinwald, H. Stoeckli-Evans. J. Organomet. Chem., 488 (1995), p. 85

[25] A.J. Deeming, M.K. Shinhmar, A.J. Arce, Y.D. Sanctis. J. Chem. Soc., Dalton Trans. (1999), p. 1153

[26] M.N. Uddin, N. Begum, M.R. Hassan, G. Hogarth, S.E. Kabir, M.A. Miah, E. Nordlander, D.A. Tocher. J. Chem. Soc., Dalton Trans. (2008), p. 6219

[27]

M.N. Uddin, M.A. Mottalib, N. Begum, S. Ghosh, A.K. Raha, D.T. Haworth, S.V. Lindeman, T.A. Siddiquee, D.W. Bennett, G. Hogarth, E . Nordlander, S.E. Kabir. Organometallics, 28 (2009), p. 1514

[28]_(a) U. Koelle. J. Organomet. Chem., 155 (1978), p. 53. (b) G.W. Harris, J.C.A. Boeyens, N.J. Coville. J. Chem. Soc., Dalton Trans. (1985), p. 2277. (c)D.R. Grad, T.L. Brown. J. Am. Chem. Soc., 104 (1982), p. 6340

[29] saint Software for CCD Diffractometer, V.7.23A, Bruker AXS, 2005.

[30] G.M. Sheldrick, sadbs-2004/1, Program for empirical absorption correction of area-detector data, Institüt für Anorganische Chemie der Universität, Göttingen, Germany, 2005. 
[31] Program XS from shelxtl package, V. 6.12, Bruker AXS, 2001.

[32] Program XL from shelxtl package, V. 6.10, Bruker AXS, 2001.

[33] M. Khatun, S. Ghosh, D.T. Haworth, S.V. Lindeman, T.A. Siddiquee, D.W. Bennett, G. Hogarth, E. Nordlander, S.E. Kabir. J. Organomet. Chem., 694 (2009), p. 2941

[34]

S.E. Kabir, F. Ahmed, S. Ghosh, M.R. Hassan, M.S. Islam, A. Sharmin, D.A. Tocher, D.T. Haworth, S.V. Lindeman, T.A. Siddiquee, D.W. Bennet, K.I. Hardcastle. J. Organomet. Chem., 693 (2008), p. 2657

[35] U. Flörke, H.J. Haupt. Z. Kristallogr., 201 (1992), p. 317 